\title{
Effect of moderate consumption of distilled $v$. fermented alcohol beverages on lymphocyte subsets
}

\author{
L. E. Díaz, J. Romeo, S. Gomez-Martínez, E. Nova and A. Marcos \\ Immunonutrition Research Group, Department of Metabolism and Nutrition, Institute of Food Science and Technology and \\ Nutrition (ICTAN), Spanish National Research Council (CSIC), Madrid, Spain
}

Diverse changes in organs and tissues as well as alterations on the immune function and the number of lymphoid cells are promoted by chronic or excessive alcohol consumption ${ }^{(1)}$. This study was aimed at evaluating the effects of moderate alcohol consumption (beer and ethanol) on lymphocyte subsets in the peripheral blood, thymus, spleen and Peyer's patches in animal model.

Forty Wistar adult male rats were divided into four groups with different beverage consumption: (1) water (control), (2) beer diluted with water, matching daily moderate alcohol consumption, (3) beer without alcohol diluted with water and (4) ethanol diluted with water, matching daily moderate alcohol consumption. The moderate ethanol intake either as beer or ethanol was $1.16 \mathrm{~g}$ alcohol $/ \mathrm{kg}$ per $\mathrm{d}$. The intervention period was 4 weeks long. Animals were killed by decapitation and the blood, thymus, spleen and Peyer's patch samples were collected. Samples were incubated with monoclonal antibodies purchased from BD Biosciences (San José, CA, USA) to differentiate $\mathrm{CD}^{+}$(Total T-cells), $\mathrm{CD}^{+}$(mature T-cells), $\mathrm{CD}^{+}$(T-helper cells), CD8 ${ }^{+}$(T-cytotoxic cells), CD45 ${ }^{+} \mathrm{RA}^{+}\left(\mathrm{B}-{ }^{-}\right.$ells) and CD161 ${ }^{+}$ (natural killer cells), and were analysed by flow cytometry (FACSCAN PLUS DUAL LASER, Becton Dickinson Sunnyvale, CA, USA).

No significant changes were observed in T-, B- and NK-cell subset percentages from thymus, spleen, Peyer's patches or peripheral blood.

These results confirm that the moderate consumption of both alcoholic beverages (beer and ethanol) do not change the percentage of lymphocyte subsets analysed both from tissues or peripheral blood (Figs. 1 and 2).

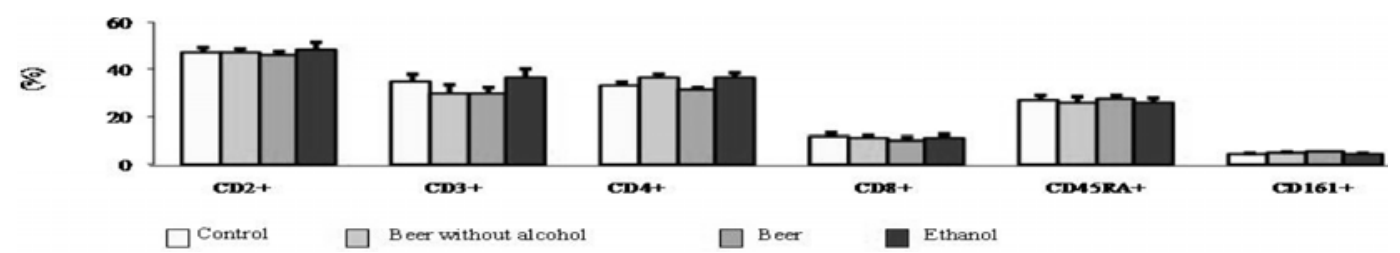

Fig. 1. Lymphocyte subsets in peripheral blood.

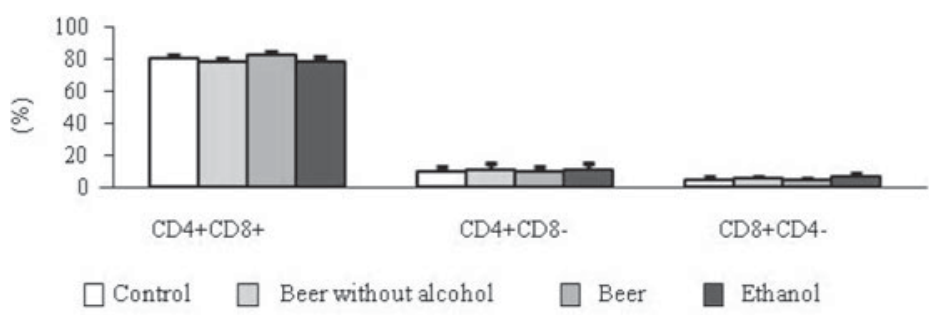

Fig. 2. Lymphocyte subsets in thymus.

We would like to acknowledge the financial support offered by Centro de Información Cerveza y Salud (Beer and Health Information Centre) from Spain.

1. Díaz LE, Montero A, González-Gross M, et al. (2002) Eur J Clin Nutr 56, S50-S53. 